\title{
INVESTIGATION OF A JEFFERY-HAMEL FLOW BETWEEN TWO RECTANGULAR INCLINED SMOOTH WALLS USING THE DIFFERENTIAL TRANSFORM METHOD
}

\author{
N.D. Patel, Ramakanta Meher \\ Department of Mathematics, S.V. National Institute of Technology \\ Surat, India \\ nirmaths@gmail.com,meher_ramakanta@yahoo.com \\ Received: 30 November 2018; Accepted: 28 February 2019
}

\begin{abstract}
In this article, the Differential Transform Method (DTM) is applied to derive a semi-analytic solution for the non-linear MHD (Magneto Hydro Dynamics) Jeffery-Hamel flow between rectangular inclined smooth planes. A non-linear ordinary differential equation of order four is obtained from Navier-Stokes equations using similar transformation. A comparison between DTM, PM (Perturbation Method) and numerical solution is shown here to validate the obtained results with its convergence analysis for different values of $\mathrm{m}$ and a Reynolds number in divergent channels.
\end{abstract}

MSC 2010: $34 A 34,34 B 60$

Keywords: Differential Transform Method, Jeffery-Hamel flow, higher order non-linear ordinary differential equation

\section{Introduction}

There is a great importance of incompressible fluid flow with heat transfer in cooling systems of malls and nuclear plants. The effect of an angle between two unparalleled solid walls meeting at a vertex on velocity of viscous fluid was studied by Hamel [1] and Jeffery [2]. They derived a mathematical formulation to explain the behaviour of a velocity profile for both divergent and convergent channels. Due to the importance of the Jeffery-Hamel flow problem in industry applications, some researchers analysed the Jeffery-Hamel problem with the effect of magnetic field, heat transfer and Nano-particle fluid flow. Akulenko et al. [3] discussed the solution of the problem of a steady viscous flow in a convergent channel by taking different values of Reynolds numbers to explain the physical phenomena of the problem. Makinde and Mhone [4] used a special type of semi-numerical Hermite-Padé approximation approach to obtain the solution of the MHD Jeffery-Hamel problem. Moghimi et al. [5] solved the MHD Jeffery-Hamel flow problem by using Homotopy Analysis Method in non-parallel sides, while the same type of problem was solved by Mustafa et al. [6] with RKHS (Reproducing Kernel Hilbert Space Method). 
Later on, the same problem was discussed by Sheikholeslami et al. [7] with the consideration of the magnetic field effect. For the first time, Mustafa [8] analyzed the Jeffery-Hamel flow problem with stretchable walls. Naveed [9] investigated a special case of the flow in which the walls were taken to be shirking and stretching. Singh and Shodiya [10] used the Modified Sumudu Transform technique to estimate the nature of Jeffery-Hamel flow while Eman et al. [11] introduced a multi-step generalized Differential Transform Method for finding the solution of a time-fractional non-linear block system. The consequence of thermal radiation on the classical Jeffery-Hamel flow problem due to the appoint sink or source term in parallel plates was analysed by Barzegar [12] for the particular case in which the plates are adjusted for stretchability. Dongochi and Divsalar [13] introduced the Duan Rach Approach (DRA) to analyze the physical phenomena arising during flow and heat transfer of MHD nano-fluid between two plates in the presence of thermal radiation. Vasile et al. [14] applied Optimal Homotopy Asymptotic Method to estimate the behaviour of MHD Jeffery-Hamel flow and showed that OHAM is not affected by large/small variation of parameters. Nagler [15-17] developed the Jeffery-Hamel flow problem of nano-fluid with the effects of wall friction and analytically and numerically solved the problem to validate results. Later he derived a mathematical formulation of Jeffery-Hamel flow with the consideration of the flow as a non-Newtonian flow. Khan et al. [18] used the Haar wavelet method and numerically investigated the Jeffery-Hamel flow and heat transfer in Eyring-Powell fluid in the presence of an outer magnetic field. Asmat, Najeeb and Farah [19] explored the Jeffery-Hamel flow of an incompressible non-Newtonian fluid inside the non-parallel walls and observed the influence of heat transfer in the flow field. Egashira et al. [20] extended the Jeffery-Hamel flow problem to elucidate characteristics of low-Re flows in a microscopic channel by taking a permeable wall. Many approaches, like the travelling wave transformation method, the Cole-Hopf transformation method, the optimal Homotopy asymptotic method, and the generalized boundary element approach were applied to solve the problems of Jeffery-Hamel flows and other fluid mechanics problems which are inherently nonlinear. In fact, in most of the cases, such problems do not have analytical solutions so there is great a importance of numerical or approximate solution of the problem to examine the nature of physical phenomena of the problems with certain limitations. The Differential Transform method (DTM) is an approximate analytical method introduced by Zhou and Pukchov [21] in 1986 to solve the problems that arise in electric circuit analysis. Later on, several authors [11,22-24] used this DTM to derive the solution of non-linear Gas Dynamics, Klein-Gordon equations arising in fluid flow problems, fuzzy partial differential equations, irrational order fractional differential equations, fractional non-linear Bloch system the thermal analysis for exponential fins under sensible and latent heat transfer, Kolmogorov-Petrovskii-Piskunov equation and for different types of Phenomena arising in fluid flow through Porous Media.

Here in this work, the Magneto Hydro Dynamics Jeffery-Hamel flow between rectangular inclined smooth planes is considered and mathematical formulation is obtained from Navier-Stokes equations with the consideration of different slopes 
and Reynolds numbers. The Differential Transform Method (DTM) is used to study the variations of velocity profiles during the fluid flow between rectangular inclined smooth planes. Finally, the obtained results have been validated by comparing the obtained results with the results obtained through the Perturbation method and the Runge-Kutta method.

Here, a Cartesian coordinates system is considered in a such a way that straight lines $y=m x+d_{0}$ and $y=-m x-d_{0}$ work as a higher plate and lower plates respectively in a rectangular unparalleled inclined smooth planes as shown in Figure 1.

\section{Mathematical formulation of the problem}

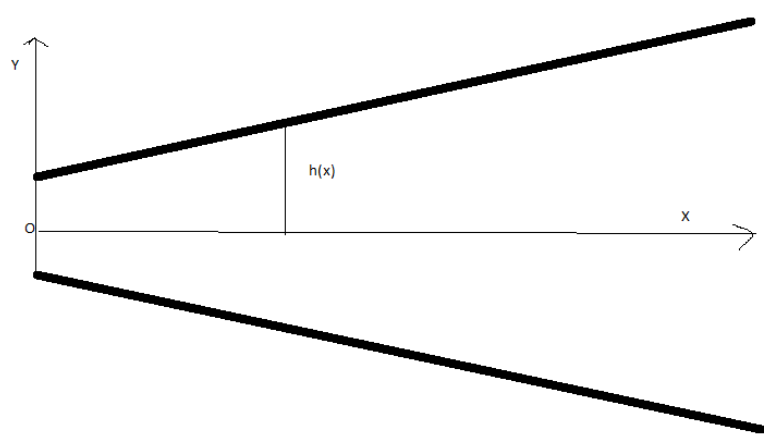

Fig. 1. Flow between plates with slope $m$

Let us consider a 2-dimensional, steady fluid flow between two inclined plates which are isolated by $2 h(x)$ distance. Without loss of generality, we use the Cartesian coordinate system in such a way that the $\mathrm{X}$-axis is along the centre line and the $\mathrm{Y}$-axis is normal to it as shown in Figure 1. Let $f(x, y)$ and $g(x, y)$ be velocity components in $x$ and $y$ directions, respectively. As $x$ is increasing, the height of wall is also increasing and the upper wall geometry for converging (diverging) channel $h(x)$ is defined by $h(x)=d_{0}+m x$ where $2 d_{0}$ is outlet/ inlet constant distance between two plates and $\mathrm{m}$ is slope of plate as shown in Figure 1. The continuity and Navier-Stokes equations are:

$$
\begin{gathered}
\frac{\partial f}{\partial x}+\frac{\partial g}{\partial y}=0 \\
f \frac{\partial f}{\partial x}+g \frac{\partial g}{\partial y}=-\frac{1}{\rho} \frac{\partial p}{\partial x}+k_{0}\left(\frac{\partial^{2} f}{\partial x^{2}}+\frac{\partial^{2} f}{\partial y^{2}}\right)
\end{gathered}
$$




$$
f \frac{\partial f}{\partial x}+g \frac{\partial g}{\partial y}=-\frac{1}{\rho} \frac{\partial p}{\partial x}+k_{0}\left(\frac{\partial^{2} g}{\partial x^{2}}+\frac{\partial^{2} g}{\partial y^{2}}\right)
$$

where $\rho$ is the the fluid density, $p$ is the pressure, $k_{0}$ is the kinematic viscosity. Define stream function $\xi(x, y)$ in such a way that it satisfies equation (1), i.e.

$$
f=\frac{\partial \xi}{\partial y}, g=-\frac{\partial \xi}{\partial x}
$$

The transformation $\xi=h(x) F(X) \phi(\gamma)$, where $\gamma=\frac{y}{h(x)}, F(X)=\frac{F_{0}}{m X+1}$ are the free stream at centre of channel and $X=\frac{x}{h_{0}}$, can be used to convert the Navier-Stokes equations into a higher order ordinary differential equation.

By comparing equation (2) and (3), it obtains a vorticity $\psi$ transport equation

$$
f \frac{\partial \psi}{\partial x}+g \frac{\partial \psi}{\partial y}=k_{0}\left(\frac{\partial^{2} \psi}{\partial x^{2}}+\frac{\partial^{2} \psi}{\partial y^{2}}\right)
$$

where $\psi=\frac{\partial g}{\partial x}-\frac{\partial f}{\partial y}$.

As per physical requirements, it is assumed that the axial velocity component $f(x, y)$ has the local maximum velocity $F(x)$ at centre of channel. Also velocity component perpendicular to $\mathrm{x}$-axis and vorticity will zero at centre line $(y=0)$ while the fluid flow is symmetrical about the centre line $x=0$ of channel. These are the following boundary conditions as discussed above:

$$
y=0: f(x, y)=F(x), \psi(x, y)=0, \xi(x, y)=0 . \quad y=h(x): f(x, y)=0
$$

Upon using the similarity transformation, the vorticity Eq. (4) reduced in to

$$
\begin{array}{r}
\left(1+m^{2} \gamma^{2}\right)^{2} \phi^{i v}+12 m^{2} \gamma\left(1+m^{2} \gamma^{2}\right) \phi^{i i i}+12 m^{2}\left(1+3 m^{2} \gamma^{2}\right) \phi^{i i} \\
+2 m \operatorname{Re}\left(1+m^{2} \gamma^{2}\right) \phi^{i} \phi^{i i}+4 m^{3} \operatorname{Re} \gamma\left(\phi^{i}\right)^{2}+24 m^{4} \gamma \phi^{i}=0
\end{array}
$$

Here, the Reynolds number is given by $R e=\frac{d_{0} F_{0}}{k_{0}}$ and it is positive and negative for diverging and converging flow respectively. Similarly, boundary conditions in Eq. (5) reduced to following exact form as:

$$
\phi(0)=0, \phi^{i}(0)=1, \phi^{i i}(0)=0 \text { and } \phi^{i}(1)=0
$$




\section{DTM for solving Ordinary Differential Equation}

Differential Transform of function $e(\gamma)$ can be defined as follows:

$$
E(k)=\frac{1}{k !}\left[\frac{d^{k} \phi(\gamma)}{d \gamma^{k}}\right]_{\gamma=0}
$$

where $e(\gamma)$ is original function and $E(k)$ is the transformed function. The uppercase and lowercase letters represent the transformed and original function respectively. The inverse differential transform of $\mathrm{E}(\mathrm{k})$ is defined as:

$$
\phi(\gamma)=\sum_{0}^{\infty} E(k) \gamma^{k}
$$

Using equation (9) in (10), it gives,

$$
\phi(\gamma)=\sum_{0}^{\infty}\left[\frac{d^{k} \phi(\gamma)}{d \gamma^{k}}\right]_{\eta=0} \frac{\gamma^{k}}{k !}
$$

\section{Solution of the MHD Jeffery-Hamel flow with heat transfer problem using Differential Transform Method}

By applying the fundamental operations of the differential transformation method to equation (6), it obtains,

$$
\begin{aligned}
& \sum_{r=0}^{k}\left\{\delta(r)+2 m^{2} \delta(r-2)+m^{4} \delta(r-4)\right\}\{(k-r+1)(k-r+2)(k-r+3) E(k-r+3)\} \\
& +\sum_{r=0}^{k}\left\{12 m^{2} \delta(r-1)+12 m^{4} \delta(r-3)\right\}\{(k-r+1)(k-r+2)(k-r+3) E(k-r+3)\} \\
& +\sum_{r=0}^{k}\left\{12 m^{2} \delta(r)+36 m^{4} \delta(r-2)\right\}\{(k-r+1)(k-r+2) E(k-r+2)\} \\
& +\sum_{r=0}^{k} \sum_{t=0}^{k-r}\left\{\left\{2 m R \delta(r)+2 R m^{3} \delta(r-2)\right\}\right. \\
& \quad \times\{(t+1) E(t+1)\}\{(k-r-t+1)(k-r-t+2) E(k-r-t+2)\}\} \\
& +4 m^{3} R \sum_{r=0}^{k} \sum_{t=0}^{k-r}\{\{\delta(r-1)\}\{(t+1) E(t+1)\}\{(k-r-t+1) E(k-r-t+1)\}\}
\end{aligned}
$$




$$
+24 m^{4} \sum_{r=0}^{k}\{\delta(r-1)\}\{(k-r+1) E(k-r+1)\}=0
$$

By solving above with conditions in eq. (7), the formula becomes,

$$
\begin{gathered}
\phi(\gamma)=\gamma+\left(-\frac{1}{3}-\frac{2 m R e}{45}+m^{2}\left(-\frac{2}{3}-\frac{163(R e)^{2}}{56700}\right)+m^{3}\left(-\frac{R e}{63}-\frac{2179(R e)^{3}}{24324300}\right)\right) \gamma^{3} \\
\left(\frac{m R e}{30}+m^{2}\left(\frac{2}{5}+\frac{R e^{2}}{225}\right)+m^{3}\left(\frac{13 R e}{150}+\frac{163 R e^{3}}{567000}\right)\right) \gamma^{5} \\
+\left(-\frac{m R e}{210}-\frac{m^{2} R e^{2}}{350}+m^{3}\left(-\frac{R e}{14}-\frac{751 R e^{3}}{1984500}\right)\right) \gamma^{7} \\
+\left(\frac{m^{2} R e^{2}}{1260}+m^{3}\left(\frac{4 R e}{315}+\frac{29 R e^{3}}{113400}\right)\right) \gamma^{9}+\left(-\frac{m^{2} R e^{2}}{14850}-\frac{191 m^{3} R e^{3}}{2079000}\right) \gamma^{11} \\
+\frac{127 m^{3} R e^{3}}{8108100} \gamma^{13}-\frac{m^{3} R e^{3}}{1053000} \gamma^{15}+\ldots
\end{gathered}
$$

which describes the velocity profiles of Jeffery Hamel flow between two inclined planes with different values of $m$ and a Reynolds number.

\section{Convergence for DTM}

Theorem: [25] Let $\phi$ be an operator from a Hilbert space $H_{0}$ in to $H_{0}$ and let $E$ be an exact solution of Eq. (6). $\sum_{i=0}^{\infty} E_{i}$ which is obtained by Eq. (8) converges to the exact solution $E$, if there exists a $\Gamma, 0 \leq \Gamma<1$, such that $\left\|E_{k+1}\right\| \leq \Gamma\left\|E_{k}\right\|, \forall k \in \mathrm{N} \cup\{0\}$. Proof: We have

$$
\begin{aligned}
& S_{0}=0, \\
& S_{1}=S_{0}+E_{1}=E_{1}, \\
& S_{2}=S_{1}+E_{2}=E_{1}+E_{2}, \\
& \quad \vdots \\
& S_{n}=S_{n-1}+E_{n}=E_{1}+E_{2}+E_{3}+\ldots+E_{n}
\end{aligned}
$$

and we will show that $\left\{S_{n}\right\}_{n=0}^{\infty}$ is a Cauchy sequence in a Hilbert Space $H_{0}$.

Now for

$\left\|S_{n+1}-S_{n}\right\|=\left\|E_{n+1}\right\| \leq \Gamma\left\|E_{n}\right\| \leq \Gamma^{2}\left\|E_{n-1}\right\| \leq \ldots \leq \Gamma^{n+1}\left\|E_{0}\right\|$ for every $n, m \in N$, 
$n \geq m$ we have

$$
\begin{aligned}
\| S_{n} & -S_{m}\|=\|\left(S_{n}-S_{n-1}\right)+\left(S_{n-1}-S_{n-2}\right)+\cdots+\left(S_{m-2}-S_{m+1}\right)+\left(S_{m+1}-S_{m}\right) \| \\
& \leq\left\|S_{n}-S_{n-1}\right\|+\left\|S_{n-1}-S_{n-2}\right\|+\cdots+\left\|S_{m-2}-S_{m+1}\right\|+\left\|S_{m+1}-S_{m}\right\| \\
& \leq \Gamma^{n}\left\|E_{0}\right\|+\Gamma^{n-1}\left\|E_{0}\right\|+\Gamma^{n-2}\left\|E_{0}\right\|+\cdots+\Gamma^{m+2}\left\|E_{0}\right\|+\Gamma^{m+1}\left\|E_{0}\right\| \\
& \leq\left(\Gamma^{m+1}+\Gamma^{m+2}+\cdots\right)\left\|E_{0}\right\| \\
& =\frac{\Gamma^{m+1}}{1-\Gamma}\left\|E_{0}\right\|
\end{aligned}
$$

Which implies $\lim _{n, m \rightarrow \infty}\left\|S_{n}-S_{m}\right\|=0$, i.e., $\left\{S_{n}\right\}_{n=0}^{\infty}$ is a Cauchy sequence in a Hilbert space $H_{0}$ and it convergence to $S$ for $S \in H_{0}$.

Definition: For every $i \in N \cup\{0\}, \Gamma_{i}$ can be defined as

$$
\Gamma_{i}=\left\{\begin{array}{c}
\frac{\left\|E_{i+1}\right\|}{\left\|E_{i}\right\|},\left\|E_{i}\right\| \neq 0 \\
0,\left\|E_{i}\right\|=0
\end{array}\right.
$$

Corollary: If $0 \leq \Gamma_{i}<1, i=1,2,3, \ldots$, then $\sum_{i=0}^{\infty} E_{i}$ is converges to the exact solution $E$.

Now by Corollary, since

$$
\Gamma_{0}=\frac{\left\|E_{1}\right\|}{\left\|E_{0}\right\|}=0<1, \Gamma_{1}=\frac{\left\|E_{2}\right\|}{\left\|E_{1}\right\|}=0<1, \Gamma_{2}=\frac{\left\|E_{3}\right\|}{\left\|E_{2}\right\|}=0<1
$$

similarly, $\Gamma_{n}=0$ for all $\mathrm{n}$. Therefore $\sum_{0}^{\infty} E(k) \gamma^{k}$ is convergent.

\section{Results and discussion}

For the validity of the obtained results, the obtained results are compared with the existing results of the Perturbation Method and Numerical results in Table 1, which can be observed that there is a good agreement between the obtained DTM results with the available results. Figures 2 and 3 discuss the variation of velocity profiles for different Reynold's number with constant slope and one can observe that a flow velocity be more for lower Reynold's number where as the flow velocity be decreases as the value of Reynold's number is increases i.e. the flow velocity is maximum for $R e=0$ and it is minimum for $R e=15$. Similarly Figures 4 and 5 discusses the variations of velocity profiles with different slopes keeping Re fixed and one can notice that a flow velocity be more in zero slope planes i.e for $m=0$ where as the flow velocity be decreases as the value of slopes is increases i.e. the flow velocity is maximum for $m=0$ while it is minimum for $m=0.9$, so overall it can be observed that there is a greater role of slopes and the Reynold's number on velocity profiles in fluid flow through inclined smooth planes. 


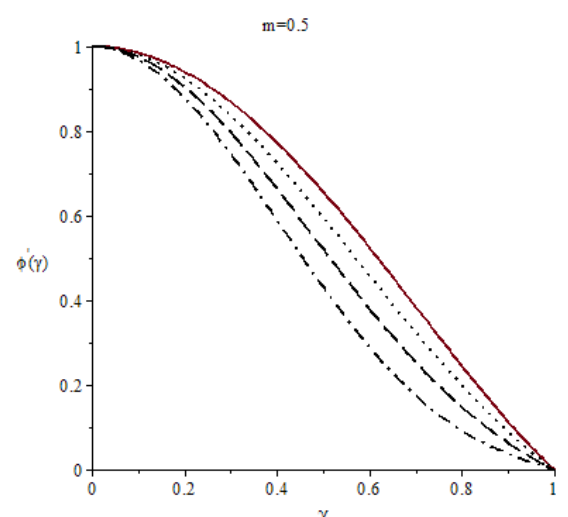

$-\operatorname{Re}=0 \cdots \cdot \operatorname{Re}=5--\operatorname{Re}=10-\cdot-\operatorname{Re}=15$

Fig. 2. Variations of velocity profiles with different $R e$ and for $m=0.5$

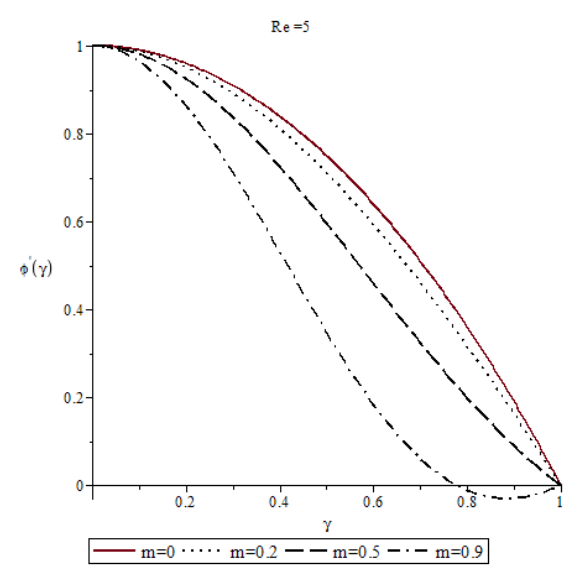

Fig. 4. Variations of velocity profiles with different slopes and for $R e=5$

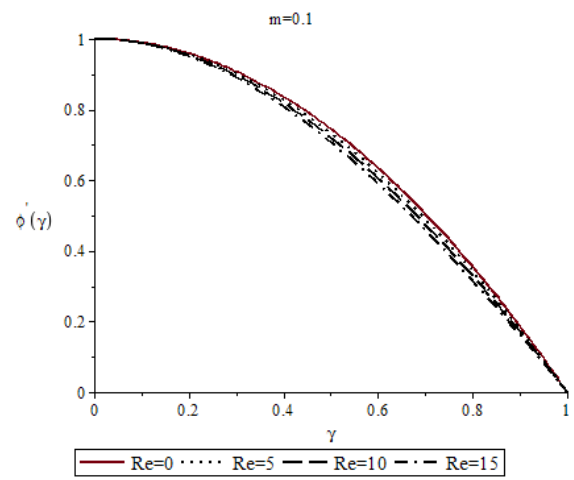

Fig. 3. Variations of velocity profiles with different $R e$ and for $m=0.1$

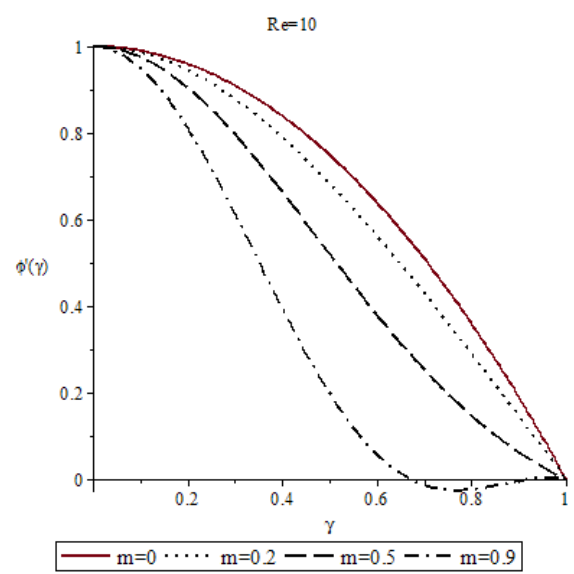

Fig. 5. Variations of velocity profiles with different slopes and for $R e=10$

Similarly, Figure 6, discusses the contours of stream lines for different values of $\mathrm{m}$ that are plotted for different slopes.

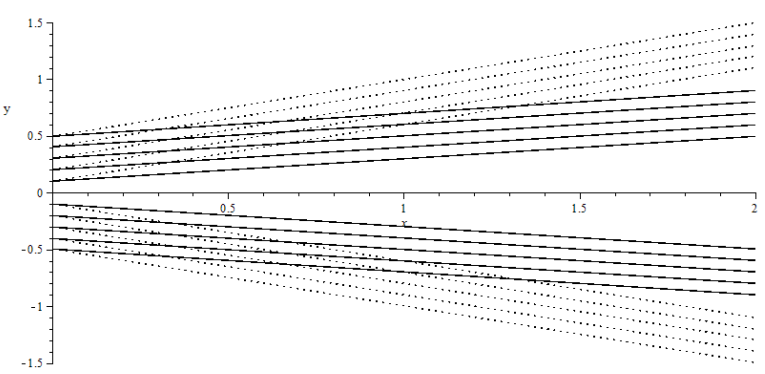

Fig. 6. Stream contours are plotted for $m=0.2$ (solid lines) and $m=0.5$ (dotted lines) 
Table 1

\begin{tabular}{|c|c|c|c|c|c|}
\hline \multicolumn{6}{|c|}{ Comparison of DTM with Peturbation Method [26] and Numerical solution } \\
\hline \multicolumn{6}{|c|}{ Error-1 = $\mid$ DTM-NM $\mid$, Error-2 $=\mid$ PM-NM $\mid$} \\
\hline $\mathbf{Y}$ & DTM & $\begin{array}{l}\text { Peturbation } \\
\text { Method }\end{array}$ & Numerical & Error-1 & Error-2 \\
\hline \multicolumn{6}{|c|}{$\operatorname{Re}=20, \mathrm{~m}=0.2$} \\
\hline 0.102 & 0.101765726 & 0.101767 & 0.101766 & 2.739E-07 & $1 \mathrm{E}-06$ \\
\hline 0.551 & 0.510338563 & 0.510382 & 0.510585 & 0.00024644 & 0.000203 \\
\hline 0.7551 & 0.642132813 & 0.642014 & 0.642788 & 0.00065519 & 0.000774 \\
\hline 0.9592 & 0.706639929 & 0.706291 & 0.707638 & 0.00099807 & 0.001347 \\
\hline \multicolumn{6}{|c|}{$\mathrm{Re}=-3, \mathrm{~m}=0.2$} \\
\hline 0.102 & 0.101645541 & 0.101646 & 0.101646 & 4.592E-07 & 0 \\
\hline 0.551 & 0.494957305 & 0.494954 & 0.494998 & $4.0695 \mathrm{E}-05$ & $4.4 \mathrm{E}-05$ \\
\hline 0.7551 & 0.610699578 & 0.610674 & 0.610847 & 0.00014742 & 0.000173 \\
\hline 0.9592 & 0.663428649 & 0.663372 & 0.663708 & 0.00027935 & 0.000336 \\
\hline \multicolumn{6}{|c|}{$\mathrm{Re}=-2, \mathrm{~m}=0.2$} \\
\hline 0.102 & 0.101636641 & 0.101637 & 0.101637 & $3.594 \mathrm{E}-07$ & 0 \\
\hline 0.551 & 0.49385821 & 0.493856 & 0.493897 & $3.879 \mathrm{E}-05$ & $4.1 \mathrm{E}-05$ \\
\hline 0.7551 & 0.608516065 & 0.608499 & 0.608658 & 0.00014193 & 0.000159 \\
\hline 0.9592 & 0.660491166 & 0.660454 & 0.660761 & 0.00026983 & 0.000307 \\
\hline \multicolumn{6}{|c|}{$\operatorname{Re}=-2, m=0.5$} \\
\hline 0.102 & 0.101518361 & 0.10152 & 0.101519 & $6.387 \mathrm{E}-07$ & 1E-06 \\
\hline 0.551 & 0.477785481 & 0.4777 & 0.4792 & 0.00141452 & 0.0015 \\
\hline 0.7551 & 0.57415105 & 0.573571 & 0.579222 & 0.00507095 & 0.005651 \\
\hline 0.9592 & 0.611359882 & 0.609895 & 0.620818 & 0.00945812 & 0.010923 \\
\hline
\end{tabular}

\section{Conclusions}

In this article, the flow behaviour of the velocity profiles in different slopes and for a different Reynold's number during fluid flow is shown between two parallel planes in a rectangular coordinate system. Here, the DTM is applied to find the solutions for the velocity profiles of the fluid flow and has validated the efficiency of the method by comparing the obtained results with the results obtained by the Perturbation Method \& Numerical results. It can be concluded that DTM is more reliable method as compared to other available methods in the study of Jeffery-Hamel flow problems.

\section{References}

[1] Hamel, G. (1917). Spiralformige bewegungen zaher flussigkeiten. Jahresbericht der Deutschen Mathematiker-Vereinigung, 25, 34-60.

[2] Jeffery, G.B. (1915). The two-dimensional steady motion of a viscous fluid. Philosophical Magazine Series, 6, 29, 455-465.

[3] Akulenko, L.D., Georgevskii, D.V., \& Kumakshev, S.A. (2004). Solutions of the Jeffery-Hamel problem regularly extendable in the Reynolds number. Fluid Dynamics, 39, 1, 12-28.

[4] Makinde, O.D., \& Mhone, P.Y. (2006). Hermite-Padé approximation approach to MHD Jeffery- 
Hamel flows. Appl. Math. Comput., 181, 966-972.

[5] Moghimi, S.M., Domairry, G., Soheil, S., Ghasemi E., \& Bararnia, H. (2011). Application of homotopy analysis method to solve MHD Jeffery-Hamel flows in non-parallel walls. Advances in Engineering Software, 4, 108-113.

[6] Mustafa, I., Akgul, A., \& Kilıçman, A. (2013). A new application of the reproducing kernel Hilbert space method to solve MHD Jeffery-Hamel flows problem in non-parallel walls. Abstract and Applied Analysis, 2013, Article ID 239454.

[7] Sheikholeslami, M., Ganji, D.D., Ashorynejad, H.R., \& Rokni, H.B. (2012). Analytical investigation of Jeffery-Hamel flow with high magnetic field and nanoparticle by Adomian decomposition method. Applied Mathematics and Mechanics, 33, 1, 25-36.

[8] Turkyilmazoglu, M. (2014). Extending the traditional Jeffery-Hamel flow to stretchable convergent/divergent channels. Computers \& Fluids, 100, 196-203.

[9] Naveed, A. (2014). Thermal radiation effects on flow of Jeffery fluid in converging and diverging stretchable channels. Neural Comput \& Applic-Springer. DOI: 10.1007/s00521-016-2831-5.

[10] Singh, J., \& Shishodia, Y.S. (2014). A modified analytical technique for Jeffery-Hamel flow using sumudu transform. J. Assoc. Arab. Univ. Basic Appl. Sci., 16, 11-15.

[11] Eman, A., Shaher, M., \& Ahmad, A. (2014). Solving the fractional nonlinear Bloch system using the multi-step generalized differential transform method. Computers and Mathematics with Applications, 68, 2124-2132.

[12] Barzegar, M.G. (2015). Investigation of thermal radiation on traditional Jeffery-Hamel flow to stretchable convergent/divergent channels. Case Studies in Thermal Engineering, 6, 28-39.

[13] Dogonchia, A.S., Divsalara, K., \& Ganjia, D.D. (2016). Flow and heat transfer of MHD nano fluid between parallel plates the presence of thermal radiation. Computers Method in Applied Mechanics and Engineering, 310, 58-76.

[14] Vasile, M., \& Remus, D. (2016). Optimal homotopy perturbation method for non-linear differential equations governing MHD Jeffery-Hamel flow with heat transfer problem. DOI: 10.1515/phys2017-0006.

[15] Nagler, J. (2016). Jeffery-Hamel flow of nano fluid influenced by wall slip conditions. Journal of Nanofluid, 5, 960-967.

[16] Nagler, J. (2016). The electroosmotic magnetohydrodynamic nano flow. Journal of Nanofluid, 5 , 898-910 (DOI:10.1166/jon.2016.1274).

[17] Nagler, J. (2017). Jeffery-Hamel flow of non-Newtonian fluid with nonlinear viscosity and wall friction. Appl. Math. Mech., 38, 6, 815-830.

[18] Khan, N., Sultan, F., Shaikh, A., Ara, A., \& Rubbab, Q. (2016). Flow and heat transfer of MHD nano fluid between parallel plates the presence of thermal radiation. AIP ADVANCES, DOI: $10.1063 / 1.4967212$.

[19] Ara, A., Khan, N., Naz, F., Raja, M., \& Rubbab, Q. (2018). Numerical simulation for JefferyHamel flow and heat transfer of micropolar fluid based on differential evolution algorithm. AIP ADVANCES (http://aip.scitation.org/toc/adv/8/1).

[20] Egashira, R., Fujikawa, T., Yaguchi, H., \& Fujikawa, S. (2018). Microscopic and low Reynolds number flows between two intersecting permeable walls. Fluid Dyn. Res. DOI:10.1088/18737005/aab578.

[21] Zhou, J.K., \& Pukhov (1986). Differential Transformation and Application for Electrical Circuits. Wuhan China: Huazhong University Press.

[22] Khudiar, A.R., Haddad, S.A.M., \& Khalaf, S.L. (2017). Restricted fractional differential transform for solving irrational order fractional differential equations. Chaos Solitons and Fractals, 101, 81-85.

[23] Kundu, B., Das, R., \& Lee, S.K. (2017). Differential Transform method for exponential fins under sensible and latent heat transfer. Procedia Engineering, 127, 284-297. 
[24] Patel, N.D., \& Meher, R. (2017). Differential transform method for solving for fingero-imbibition phenomena arising in double phase flow through homogeneous porous media. Mathematical Sciences, IMRF Journals, 6, 1, 1-5.

[25] Patel, N.D., \& Meher, R. (2016). Analytical investigation of Jeffery-Hamel flow by modified Adomian Decomposition Method. Ain Shams Engineering Journal.

[26] Khan, M., Aamir, A., \& Asghar S. (2017). Flow between two rectangular inclined plane walls. Chinese Journal of Physics, 55, 1195-1201. 